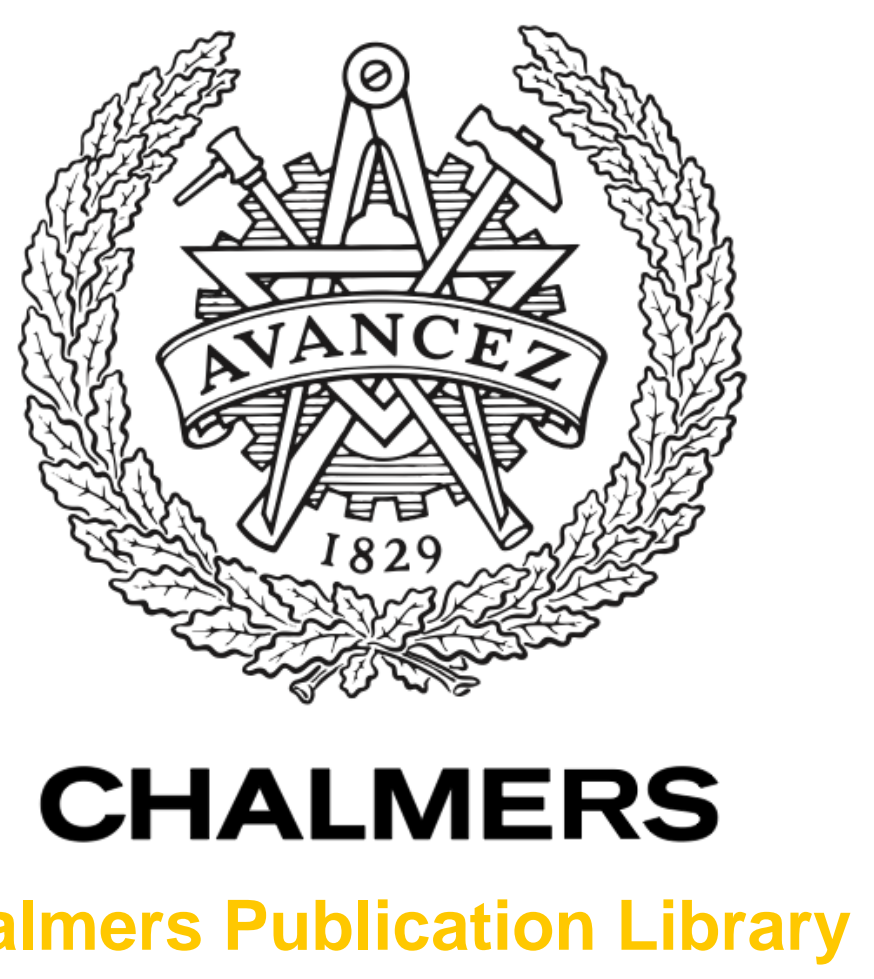

Chalmers Publication Library

\author{
Effects of Mutual Coupling on LTE MIMO Capacity for Monopole Array: \\ Comparing Reverberation Chamber Tests and Drive Tests
}

This document has been downloaded from Chalmers Publication Library (CPL). It is the author's version of a work that was accepted for publication in:

IEEE Antennas and Wireless Propagation Letters (ISSN: 1536-1225)

Citation for the published paper:

Song, H. ; Bekaryan, A. ; Schaffner, J. et al. (2015) "Effects of Mutual Coupling on LTE MIMO Capacity for Monopole Array: Comparing Reverberation Chamber Tests and Drive Tests". IEEE Antennas and Wireless Propagation Letters, vol. 14 pp. 454-457.

http://dx.doi.org/10.1109/LAWP.2014.2367453

Downloaded from: http://publications.lib.chalmers.se/publication/213938

Notice: Changes introduced as a result of publishing processes such as copy-editing and formatting may not be reflected in this document. For a definitive version of this work, please refer to the published source. Please note that access to the published version might require a subscription.

Chalmers Publication Library (CPL) offers the possibility of retrieving research publications produced at Chalmers University of Technology. It covers all types of publications: articles, dissertations, licentiate theses, masters theses, conference papers, reports etc. Since 2006 it is the official tool for Chalmers official publication statistics. To ensure that Chalmers research results are disseminated as widely as possible, an Open Access Policy has been adopted.

The CPL service is administrated and maintained by Chalmers Library. 


\title{
Effects of Mutual Coupling on LTE MIMO Capacity for Monopole Array: Comparing Reverberation Chamber Tests and Drive Tests
}

\author{
Hyok J. Song, Member, IEEE, Arthur Bekaryan, Member, IEEE, James H. Schaffner, Senior Member, \\ IEEE, Ahmed Hussain, and Per-Simon Kildal, Fellow, IEEE
}

\begin{abstract}
The roof of an automobile would appear to be an ideal platform to place MIMO antennas, having a large ground plane with plenty of space to separate antennas for optimum embedded element radiation efficiency. However, styling concerns often require that the antennas are to be placed close together. Thus, mutual coupling plays an important role in the performance of MIMO antenna system as it affects the embedded element efficiency and the channel correlation. In this letter, we present a comparison between the measured mutual coupling effect on the ergodic channel capacity of a $2 \times 2$ MIMO system in reverberation chamber (i.e. Rayleigh channel) and in a suburban outdoor environment (i.e. Rician channel) at Long Term Evolution (LTE) radio band 13. The outdoor MIMO capacity was obtained during a vehicle drive test using commercial over-the-air (OTA) LTE test equipment. All measurements were conducted with two element monopole antennas on a 24" diameter circular ground plane. The impact that mutual coupling has in the degradation of the embedded element radiation efficiency and increased correlation between the antennas, and ultimately the decrease in ergodic MIMO channel capacity is highlighted by these measurements. In addition, the results provide insight into the differences of channel capacity obtained in the rich isotropic multipath environment of a reverberation chamber and the channel capacity obtained in a real world suburban environment.
\end{abstract}

Index Terms-Antennas, antenna pattern, channel, ergodic capacity, isotropic pattern, k-factor, MIMO, monopole, mutual coupling, over-the-air, vehicle antennas, Rayleigh, reverberation chamber, Rician.

\section{INTRODUCTION}

$M$ ULTIPLE-INPUT-MULTIPLE-OUTPUT (MIMO) ANTENNA technology promises an increase in spectral efficiency and channel capacity of wireless communication systems through spatial multiplexing of data streams by employing multiple antennas at both ends of the communication link. It has been recently adopted as a key standard component for LTE (Long-Term Evolution) and WiMAX (Wireless Interoperability for Microwave Access) radio systems. Results

This paragraph of the first footnote will contain the date on which you submitted your paper for review.

H. J. Song, A. Bekaryan and J. Schaffner are with HRL Laboratories, LLC., Malibu, CA 90265 USA (e-mail: hjsong@ hrl.com).

A. Hussain and P.-S. Kildal are with Chalmers University of Technology, Gothenburg, Sweden. (e-mail: per-simon.kildal@chalmers.se). of both analytical and experimental studies on many aspects of MIMO technology have been reported in a number of reports, a few given by [1-5]. LTE service is rapidly rolling out around the world and will be included in telematics services for automobiles.

It has been well known that mutual coupling between the multiple antenna elements has a significant effect on radiation properties and spatial correlation of the antennas which could directly contribute to the achievable maximum capacity of the MIMO system [2, 6, 7]. The further the separation of the antennas, the less impact mutual coupling has on throughput.

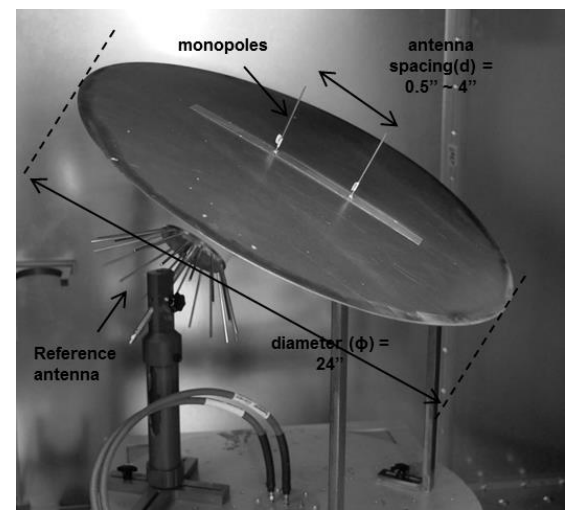

Fig. 1. Reverberation chamber measurement setup showing two identical monopoles on a 24 " diameter circular ground plane and a reference calibration antenna mounted on a pedestal.

It would appear that an automobile would be an ideal platform for MIMO antennas, with plenty of roof space to separate the antennas to eliminate mutual coupling, minimize spatial correlation, and optimize the total embedded radiation efficiency $\left(\mathrm{e}_{\mathrm{emb}}\right)$, which is defined in [8]. A more general definition in terms of S-parameters can be found in [9]. However, design and economic requirements drive MIMO antennas to be spaced close together, and mutual coupling between antennas becomes a concern. Thus it is important to understand the design trade-offs between capacity and antenna spacing for rooftop mounted antennas, especially in a real vehicle operating environment.

Although it is feasible to simulate performance of a MIMO antenna system on a vehicle in a complex electromagnetic environment, a successful performance evaluation requires embedded element patterns at a vehicle level which may not be 
easily modeled. In this case, experimental measurements of MIMO antennas to understand expected received information capacity is still needed. Recent advancements in the antenna measurement technology allow several options for characterizing the MIMO antennas. A reverberation chamber (RC) provides one of such measurement options which can efficiently characterize the MIMO antennas in terms of the embedded element efficiency, diversity gain, and the MIMO capacity in a repeatable rich isotropic multipath (RIMP) environment. The RIMP environment has the characteristic that the channels will represent the theoretical independent identically distributed (i.i.d) case if the MIMO antennas have uncoupled ports with $100 \%$ radiation efficiency. The RIMP environment is useful for characterizing the MIMO antennas on handheld user equipment (UE) since random orientation of the UE introduced by a user in a real operational scenario effectively makes the received signals at the terminal to be close to Rayleigh distributed [8, 10]. If we, however, consider the MIMO terminal antennas on an electrically large platform such as automobiles and trains in which the orientation of the antenna is relatively fixed, then the RIMP environment may no longer represent the relevant operational environment of such terminal antennas. There could be a strong direct signal component and the multipath environment would appear to have more of a Rician distribution [11]. In this case, Over-The-Air (OTA) measurements along designated routes using a LTE signal scanner could be used to assess the corresponding ergodic MIMO capacity.

In this paper, we systematically examine the mutual coupling effect on the ergodic capacity of two elements MIMO antenna on an electrically large ground plane in the ideal RIMP environment of a Bluetest ${ }^{\circledR}$ reverberation chamber and also in a real suburban operating environment using a Rohde \& Schwarz ${ }^{\circledR}$ TSMW universal radio network analyzer with drive test software. A comparison of the measurement results will be presented that point up the potential differences in MIMO capacity and the effect of mutual coupling for vehicle mounted MIMO antennas due to the different multipath environments.

\section{ANTENNA CONFIGURATION AND MEASUREMENTS}

\section{A. Antenna Configuration}

We have chosen two identical quarter wavelength monopoles for conducting the mutual coupling study in the downlink frequencies of LTE band $13(746 \sim 756 \mathrm{MHz})$ since radiation property of the monopole is well understood and it can be easily fabricated. The monopole antennas are mounted on a circular ground plane with a diameter of 24 " (i.e. $1.524 \lambda_{0}$ at $750 \mathrm{MHz}$ ) which has a sliding slot for adjusting a distance between the antennas. The two element MIMO antenna terminal, which includes the electrically large ground plane, as shown in Fig. 1, is examined for the mutual coupling and the ergodic capacity at various antenna spacing of 0.5 ", 1.0 ", 2.0", 3.0 " and 4.0 " (i.e., respectively equivalent to $0.03175,0.0635$, $0.127,0.1905$ and $0.254 \lambda_{0}$ at $750 \mathrm{MHz}$ ) in the reverberation chamber and in the suburban outdoor environment.

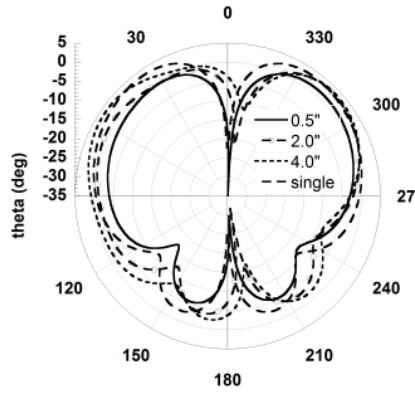

(a)

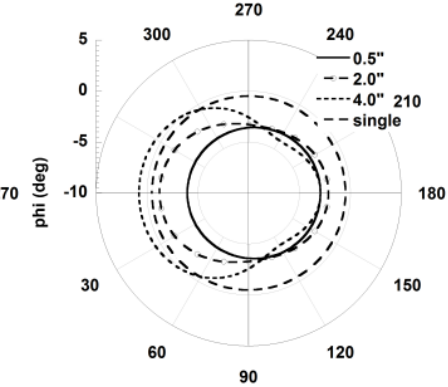

(b)
Fig. 2. Simulated embedded radiation patterns of the left monopole excited while the other terminated for the antenna spacing of 0.5 ", 2.0" and 4.0", and simulated patterns of a single monopole: (a) elevation patterns at $\phi=0^{\circ}$, and (b) azimuth patterns at $\theta=90^{\circ}$.

\section{B. Mutual Coupling and Total Radiation Efficiency}

It is well known that a presence of mutual coupling between MIMO antennas in a Rayleigh fading channel will have a negative effect on the embedded radiation efficiency, correlation and the channel capacity [6-8]. Fig. 2 shows simulated 2D embedded radiation pattern of the MIMO antenna under the test with the left monopole excited while the other terminated, in comparison to the simulated pattern of a single monopole antenna. A reduced gain of the embedded radiation pattern can be clearly seen as the antenna spacing decreases to $0.5 "$.
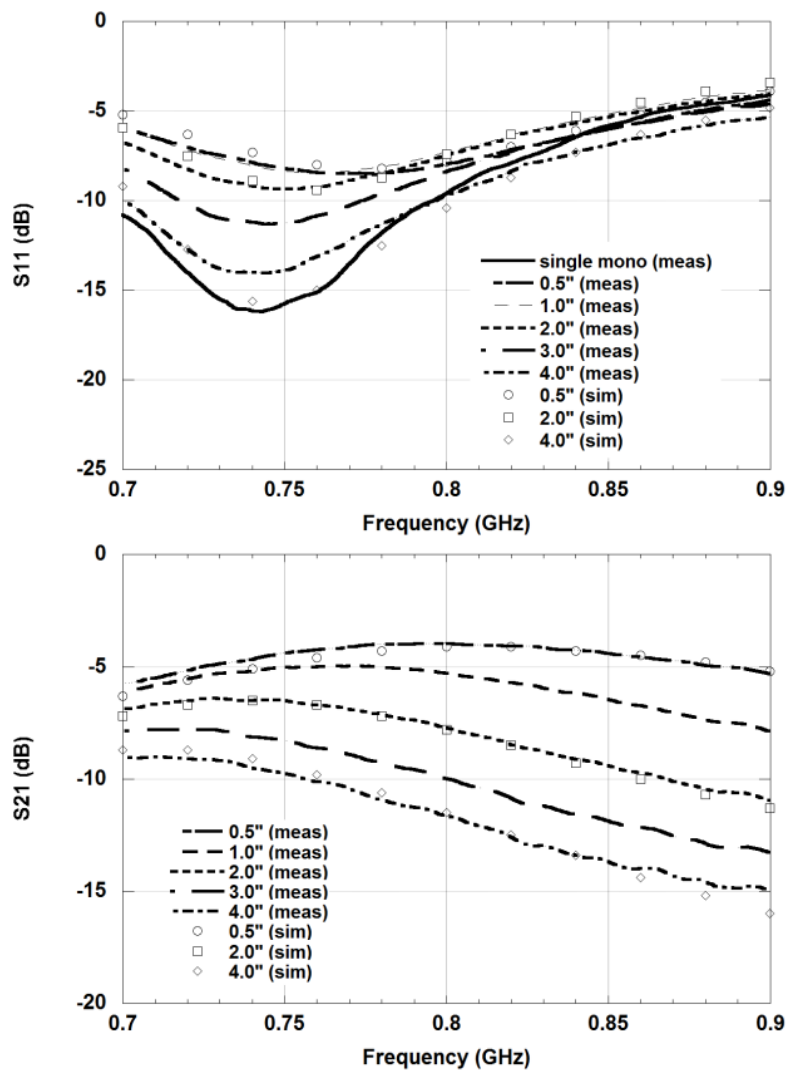

Fig. 3. Measured and simulated return loss of one of two identical monopoles (upper graph), and measured and simulated mutual coupling between two monopoles (lower graph) as the antenna spacing varied from 0.5 " to 4.0 ". 
This result is as expected since the mutual coupling gets stronger as the two antennas are placed closer and modifies the terminal impedance of the antennas that result in the degradation in the impedance matching and the total embedded radiation efficiency [8]. The total embedded radiation efficiency $\left(\mathrm{e}_{\mathrm{emb}}\right)$ for a lossless antenna is simply given by the decoupling efficiency in [9] for the single port no. 1,

$$
e_{e m b}=1-\left|S_{11}\right|^{2}-\left|S_{21}\right|^{2}
$$

, where $S_{11}$ and $S_{21}$ are the $S$-parameters of the antenna.

Fig. 3 shows measured and simulated return loss $\left(S_{11}\right)$ and mutual coupling $\left(\mathrm{S}_{21}\right)$ of the MIMO antennas under the test when the antenna spacing is varied from 0.5 " to 4.0 " and it confirms the negative effect of the mutual coupling on the impedance matching.

The total embedded radiation efficiency $\left(\mathrm{e}_{\mathrm{emb}}\right)$ and envelope correlation ( $\rho_{\text {env }}$ ) of the antenna were measured in the reverberation chamber. Fig. 4 compares the $e_{\text {emb }}$ and $\rho_{\text {env }}$ of each of the monopoles measured in the reverberation chamber along with the simulated results at $750 \mathrm{MHz}$ obtained from a full wave method of moment (MoM) based electromagnetic simulation software. The two results compare very well and it confirms the mutual coupling effect on the $e_{\mathrm{emb}}$ and $\rho_{\mathrm{env}}$ as the antenna spacing varied.

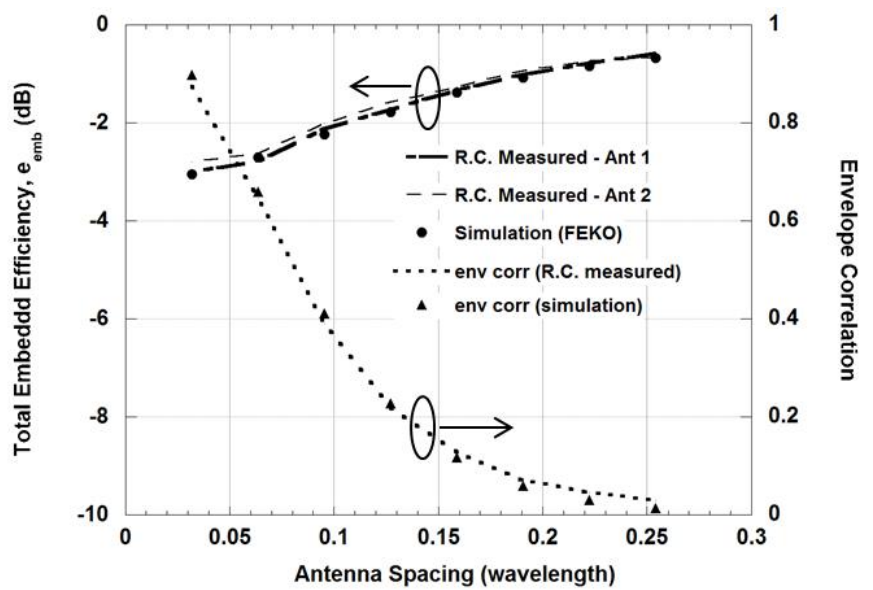

Fig. 4. Comparison of the total embedded radiation efficiency $\left(\mathrm{e}_{\mathrm{emb}}\right)$ and envelope correlation $\left(\rho_{\text {env }}\right)$ of the monopoles measured in the reverberation chamber and that of the full-wave EM simulation (FEKO).

\section{Ergodic Channel Capacity of $2 \times 2$ MIMO Antennas}

The reverberation chamber used for the measurements described here has three wideband monopoles for MIMO transmit antennas. These antennas are mounted orthogonally to each other on a cube shape metal ground plane, thus minimizing mutual coupling on the transmit side of the system. As our investigation considers a channel capacity of a $2 \times 2$ MIMO system, only two of the transmit antennas are used in the post processing of the measured channels. Although using a particular combination of two transmit antennas may create a slight polarization imbalance, the channel environment created is isotropic enough, leading to a similar result with a different two element transmit antenna combination, as shown in Fig. 5. The receive MIMO monopoles were mounted in the chamber as shown in Fig. 1. Measured complex $S_{21}$ values between each of the two transmit and receive antennas form entries of the $2 \times 2$ channel transfer matrix $\mathbf{H}$, which can be described as [ $\left.\mathbf{h}_{\mathrm{ij}}\right]$, where $h_{i j}$ represents a sub-channel gain between the $j^{\text {th }}$ transmit antenna and $i^{\text {th }}$ receive antenna. The channel matrix is normalized to a reference power level obtained from the reference antenna with a known total radiation efficiency [12], which is also labeled in Fig. 1.

An ensemble of the normalized channel matrices $\tilde{\mathbf{H}}$ was obtained for 400 measured samples per frequency at different combinations of the mode stirrer position in the RC. As the transmitter does not have the channel state information from the receiver, the power is equally transmitted from the transmit antenna elements. For this situation, a theoretical maximum channel capacity $\left(\mathrm{C}_{\text {MIMO }}\right)$ of the MIMO system can be calculated in bits/second/Hz (bps/Hz) for a given signal to noise ratio of SNR by (2) as in [13]

$$
C_{\text {MIMO }}=\log _{2}\left[\operatorname{det}\left(\mathbf{I}+\frac{S N R}{n_{t}} \mathrm{HH}^{*}\right)\right]
$$

, where $\mathbf{I}$ is the identity matrix with the size given by a minimum number of transmit antennas $\left(\mathrm{n}_{\mathrm{t}}\right)$ and receive antennas $\left(n_{r}\right)$, and $\tilde{\mathbf{H}}$ represents the normalized $n_{r} \times n_{t}$ channel matrix with the $*$ symbol denotes the conjugate transpose. The ergodic capacity of the $2 \times 2$ MIMO system is calculated for a given average SNR by averaging the capacity values obtained from all the measured channel matrices using (2). The capacity values obtained in the RC represents the channel capacity of the MIMO system in the ideal Rayleigh fading environment.

For the drive test measurements of the MIMO channel capacity, the circular ground plane with the two monopoles was mounted on a center of a passenger sedan roof. The LTE signals from available eNodeB were measured through the two receive monopole antennas simultaneously. The complex channel matrix was measured and stored for each of 50 resource blocks for the $10 \mathrm{MHz}$ bandwidth of the LTE band 13 per each measurement sample while driving over a predefined test route of approximately 11 miles in a suburban area of Los Angeles in California. The total number of the measured samples was approximately 2000 with a sampling rate of 900 milliseconds. The ergodic capacity is obtained using (2) with the SNR replaced by the total embedded radiation efficiency corrected SNR ( $\mathrm{SNR}_{\text {tot_emb}}$ ) at the antenna port after normalizing the measured channel matrices according to

$$
\mathrm{H}=\frac{\sqrt{n_{t} n_{r}}}{\|\mathrm{H}\|_{F}} \mathrm{H}
$$

, where $\|\mathbf{H}\|_{\mathrm{F}}$ denotes the Frobenius norm of the channel matrix $[12,14]$. The square of $\|\tilde{\mathbf{H}}\|_{\mathrm{F}}$ is then $\mathrm{n}_{\mathrm{r}} \times \mathrm{n}_{\mathrm{t}}$ which is equivalent to the trace of $\tilde{\mathbf{H}} \tilde{\mathbf{H}}^{*}$ and can be interpreted as the total power gain of the sub-channels [15]. The drive tests were repeated with the same antenna spacing of $0.5 ", 1.0 ", 2.0 ", 3.0$ " and 4.0" used in the reverberation chamber tests, and their corresponding ergodic channel capacities were obtained for a given average SNR. We could also have normalized to a single vertical monopole antenna with a known efficiency located at the same vehicle and uncoupled to the MIMO antenna under test, but the present normalization in (3) is equivalent to the case when the SNR in (2) is reduced by the total embedded radiation efficiency. 


\section{RESULTS AND DISCUSSION}

The ergodic channel capacities of the $2 \times 2$ MIMO system obtained from the reverberation chamber and the OTA drive test measurements were compared for a SNR of $10 \mathrm{~dB}$ as a function of the antenna spacing as shown in Fig. 5. For comparison, the simulated capacity values are also plotted in Fig. 5 for the ideal uncoupled antenna case as well as the SISO case, which assumes the antenna having isotropic radiation pattern in the independent, identically distributed fading channel. Fig. 5 clearly shows that the channel capacity measured in the RC increases and approaches the simulated ideal uncoupled case as the antenna spacing increases due to the reduction in mutual coupling. In the small antenna spacing region, the capacity of the two monopole MIMO system approaches that of SISO, but the difference in MIMO capacity increases to approximately $6.7 \%$ as the antenna spacing increases to $0.254 \lambda_{0}$. This is expected because the real world radio propagation environment where the OTA drive measurements were taken becomes more Rician, having a larger distribution of direct line-of-site component, than the ideal RIMP environment in the RC. The slightly larger OTA drive test data in the small antenna spacing region could be due to the non-uniform distribution of multipath angular spread in the drive test environment, resulting in possibly more multipath components in the higher gain angular sector of the antennas.

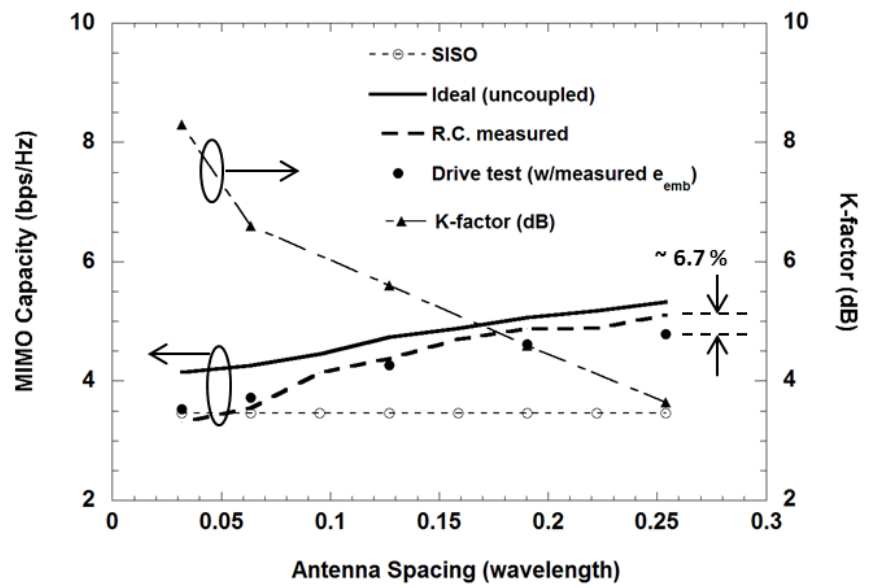

Fig. 5. Comparison of $2 \times 2$ MIMO capacity (for $S N R=10 \mathrm{~dB}$ ) of two identical monopoles on the 24 inch diameter circular ground plane, measured in the reverberation chamber and an outdoor suburban field. The K-factor estimated from the measured channel samples was also plotted.

The Rician K-factor of the MIMO channel was estimated from the OTA drive test measured samples using the statistical distribution fitting tool available in MATLAB, and is plotted for the different antenna spacing in Fig. 5 [16]. The estimated K-factor value was 8.3 (i.e. $9.2 \mathrm{~dB}$ ) for the 0.5 " (i.e. $0.032 \lambda_{0}$ at $750 \mathrm{MHz}$ ) antenna spacing and it decreases to 3.6 (i.e. $5.6 \mathrm{~dB}$ ) when the spacing increases to 4 " (i.e. $0.254 \lambda_{0}$ at $750 \mathrm{MHz}$ ).

\section{CONCLUSION}

Both the reverberation chamber and the OTA drive test measurements have their place in evaluating the performance of MIMO antennas and systems. The reverberation chamber is especially useful for quick MIMO radio characterization and radio-to-radio comparative measurement in an ideal RIMP environment. OTA drive testing is more involved and takes a longer time to collect data, however it is still essential for determining antenna placement on a vehicle and for comparative antenna testing in a non-ideal real world radio environment. With both measurement techniques the results presented in this paper show that for a $2 \times 2$ MIMO system the mutual coupling between antennas caused reduction of total embedded radiation efficiency and increase in correlation resulting in a decrease in ergodic MIMO capacity. These results can start to form the basis of general guidelines for the design and placement of $2 \times 2$ MIMO automotive antennas.

\section{REFERENCES}

[1] D. Pinchera, J. Wallace, M. D. Migliore, and M. Jensen, "Experimental analysis of a wideband adaptive-MIMO antenna," IEEE Trans. Antennas Propag., vol.56, no. 3, pp.908-913, Mar. 2008.

[2] X. Liu and M. E. Bialkowski, "Effect of antenna mutual coupling on MIMO channel estimation and capacity," International Journal of Antennas and Propag., vol. 2010, Article ID 306173, 9 pages, 2010.

[3] G. D. Durgin, Space-Time Wireless Channels. Upper Saddle River, NJ: Prentice Hall PTR, 2003.

[4] H. T. Hui, "Influence of antenna characteristics on MIMO systems with compact monopole arrays," IEEE Antennas Wireless Propag. Lett., vol. 8, pp. 133-136, 2009.

[5] M. Rumney, R. Pirkl, M. H. Landmann, and D. A. Sanchez-Hernandez, "MIMO Over-The-Air Research, Development, and Testing," International Journal of Antennas and Propag., vol. 2012, Article ID 467695, 8 pages, 2012.

[6] Z. Li, Z. Du, M. Takahashi, K. Sato, and K. Ito, "Reducing mutual coupling of MIMO antennas with parasitic elements for mobile terminals," IEEE Trans. Antennas Propag., vol. 60, no. 2, pp. 473-481, Feb. 2012.

[7] P.-S. Kildal and K. Rosengren, "Correlation and capacity of MIMO systems and mutual coupling, radiation efficiency, and diversity gain of their antennas: simulations and measurements in a reverberation chamber," IEEE Commun. Mag., vol. 42, pp. 104-112, Dec. 2004

[8] K. Rosengren and P.-S. Kildal, "Radiation efficiency, correlation, diversity gain and capacity of a six-monopole antenna array for a MIMO system: theory, simulation and measurement in reverberation chamber," IEE Proc. Microw. Antennas Propag., vol. 152, no. 1, pp.7-16, Feb. 2005.

[9] M. Ng Mou Kehn, M. V. Ivashina, P.-S. Kildal and R. Maaskant, "Definition of unifying decoupling efficiency of different array antennas case study of dense focal plane array feed for parabolic reflector", AEUE-Int. Journal of Electronics and Communications, 4 June 2009.

[10] P.-S. Kildal and J. Carlsson, "New approach to OTA testing: RIMP and pure-LOS reference environments and a hypothesis," in Proc. $7^{\text {th }}$ European Conf. on Antennas and Propag. (EuCAP), pp. 315-318, April 2013.

[11] R. Vaughan, and J. B. Andersen, Channels, Propagation and Antennas for Mobile Communications, The Institute of Electrical Engineers, London, UK, 2003.

[12] X. Chen, P.-S. Kildal, J. Carlsson, and J. Yang, "Comparison of ergodic capacities from wideband MIMO antenna measurements in reverberation chamber and anechoic chamber," IEEE Antennas Wireless Propag. Lett., vol. 10, pp. 446-449, 2011

[13] G. J. Foschini, Jr., "Layered space-time architecture for wireless communication in a fading environment when using multi-element antennas," Bell Labs Syst. Tech. J., pp. 41-59, 1996.

[14] S. Loyka and G. Levin, "On physically-based normalization of MIMO channel matrices," IEEE Trans. Wireless Commun., vol. 8, no. 3, pp. 1107-1112, Mar. 2009.

[15] A. Paulraj, D. A. Gore, R. Nabar, "ST channel and signal models," in Introduction to space-time wireless communications, Cambridge: Cambridge University Press, 2003, pp. 32-62.

[16] V.R. Anreddy and M.A. Ingram, "Capacity of measured Rician and Rayleigh indoor MIMO channels at $2.4 \mathrm{GHz}$ with polarization and spatial diversity," in Proc. 2006 IEEE Wireles Communications and Networking Conf (WCNC)., vol. 2, pp. 946-951, April 2006. 